\title{
Glomerular Filtration Dysfunction is Associated with Cardiac Adverse Remodeling in Menopausal Diabetic Chinese Women
}

This article was published in the following Dove Press journal:

Clinical Interventions in Aging

\author{
Xing Song' \\ Gang Li $\mathbb{D}^{\prime}$ \\ Yuqi Zhu' \\ Jari A Laukkanen ${ }^{2-4}$ \\ 'Division of Cardiology, Department of \\ Geriatrics, The First Affiliated Hospital of \\ Chongqing Medical University, \\ Chongqing, People's Republic of China; \\ ${ }^{2}$ Institute of Public Health and Clinical \\ Nutrition, University of Eastern Finland, \\ Kuopio, Finland; ${ }^{3}$ Institute of Clinical \\ Medicine, Department of Medicine, \\ University of Eastern Finland, Kuopio, \\ Finland; ${ }^{4}$ Central Finland Health Care \\ District, Department of Medicine, \\ Jyväskylä, Finland
}

Background: Previous studies have showed that nephropathy was associated with cardiac structural changes and dysfunction among diabetic adults. However, information on the association of glomerular filtration dysfunction with the cardiac adverse remodeling is still limited in menopausal diabetic women. Therefore, we investigated whether impaired glomerular filtration function is associated with the cardiac adverse remodeling in menopausal Chinese women with type 2 diabetes mellitus (DM).

Methods: A total of 1231 hospitalized menopausal Chinese women with type 2 DM were collected retrospectively. The cross-sectional data of echocardiography were compared among estimated glomerular filtration rate (eGFR) categorized groups.

Results: In menopausal diabetic women, moderate to severe glomerular filtration dysfunction (eGFR $<60 \mathrm{~mL} / \mathrm{min}$ per $1.73 \mathrm{~m}^{2}$ ) was found to be associated with enlarged left-side atrioventricular chambers, increased ventricular wall thickness, decreased cardiac function and dilated right ventricle (All $P<0.05$ ).

Conclusion: Glomerular filtration dysfunction is associated with cardiac adverse structural remodeling and dysfunction in menopausal Chinese women with type 2 DM.

Keywords: glomerular filtration rate, type 2 diabetes mellitus, cardiac remodeling, menopausal women, aging

\section{Introduction}

It is well known that patients with type 2 diabetes mellitus (DM) are susceptible to increased risk of cardiomyopathy, coronary atherosclerosis and cardiac death. ${ }^{1,2}$ Recent studies showed that the increased incidence of cardiovascular events and mortality in diabetic patients is also ascribed to diabetic nephropathy., ${ }^{3,4}$ Nevertheless, there are still few investigations to report that decreased estimated glomerular filtration rate (eGFR) is associated with cardiac structural changes and dysfunction in diabetic patients. ${ }^{5,6}$ Meanwhile, previous studies have found that postmenopausal women with type 2 DM had a higher risk of cardiovascular events and mortality than those of diabetic men at the same age. ${ }^{7,8}$ However, there is still limited information on whether the diabetic nephropathy was involved in cardiac adverse structural changes and dysfunction in the menopausal diabetic women. Therefore, this study explored the relationship between impaired glomerular filtration function and cardiac adverse remodeling in menopausal Chinese women with type 2 DM.
Division of Cardiology, Department of Geriatrics, The First Affiliated Hospital of Chongqing Medical University, No. I Youyi Road, Yuzhong District, Chongqing, 400016, People's Republic of China

Tel +862389011518

Fax +8623688II487

Email ganglicqmu@126.com 


\section{Methods}

\section{Patients}

At department of endocrinology in our hospital, a total of 1231 naturally menopausal Chinese women with type 2 DM were collected retrospectively by review of medical records during 2014 to 2018 in a cross-sectional study. Type 2 DM was diagnosed if fasting plasma glucose (FPG) $\geq 7.0 \mathrm{mmol} / \mathrm{L}$ and/or oral $75 \mathrm{~g}$ glucose post-loaded $2-$ hour plasma glucose $\geq 11.1 \mathrm{mmol} / \mathrm{L}$, and the plasma insulin level was normal or increased, or the subjects have been treated with hypoglycemic drugs or lifestyle modifications. ${ }^{9}$ Normal eGFR is defined as eGFR $\geq 90 \mathrm{~mL} / \mathrm{min}$ per $1.73 \mathrm{~m}^{2}$. The degree of declined eGFR is usually used to assess the severity of nephropathy (mild, $60 \leq \mathrm{eGFR}<90$; moderate, $30 \leq \mathrm{eGFR}<60$; and severe, eGFR $<30 \mathrm{~mL} / \mathrm{min}$ per $1.73 \mathrm{~m}^{2}$ respectively). ${ }^{10}$

Hypertension was confirmed if subjects had average systolic blood pressure (SBP) $\geq 140 \mathrm{mmHg}$ and/or diastolic blood pressure (DBP) $\geq 90 \mathrm{mmHg}$ at upper right arm after 10 minutes rest, or received antihypertensive drugs. Coronary heart disease was considered if myocardial infarction evidences on electrocardiograms, or angiography confirmed atherosclerotic stenosis $\geq 50 \%$ in one of coronary artery lumens, or a history of coronary stent implantation were confirmed. Stroke criteria included evidences on computed tomograms or magnetic resonance imaging. ${ }^{11}$ All patients were examined by color doppler echocardiography. We excluded patients with acute renal insufficiency, glomerulonephritis, autoimmune or infectious nephropathy, rheumatic or degenerative cardiac valvular diseases, congenital heart disease, idiopathic cardiomyopathy, atrial fibrillation, malignant tumors.

This study was conducted in accordance with the Declaration of Helsinki and approved by the ethics committee of the First Affiliated Hospital of Chongqing Medical University (No. 2020-305). The ethics committee waived the need for patients' written informed consent because of anonymous nature of the clinical data acquired retrospectively.

\section{Calculation of Glomerular Filtration Rate and Body Mass Index}

Serum creatinine $(\mathrm{Scr})>62 \mu \mathrm{mol} / \mathrm{L}, \mathrm{eGFR}=144 \times(\mathrm{Scr} \mu \mathrm{mol} /$ $\mathrm{L} \div 88.4 \div 0.7)-1.209 \times 0.993^{\text {age }}$; Scr $\leq 62 \mu \mathrm{mol} / \mathrm{L}, \mathrm{eGFR}=144$

$\times(\operatorname{Scr} \mu \mathrm{mol} / \mathrm{L} \div 88.4 \div 0.7)-0.329 \times 0.993{ }^{\text {age }} \cdot{ }^{10}$ Body mass index $(\mathrm{BMI})=$ body weight $(\mathrm{kg}) /(\text { height }(\mathrm{m}))^{2}$.

\section{Determination of Clinical Biochemistry}

Glucose was measured by hexokinase. Triglyceride (TG) and total cholesterol (TC) were measured by enzyme colorimetry. High-density lipoprotein cholesterol (HDL-C) was measured by homogeneous enzyme colorimetry. Lowdensity lipoprotein cholesterol was calculated by TC HDL-C - (TG/2.19). ${ }^{11}$

\section{Measurement of Cardiac Structure and Function}

Right atrial diameter (RAD), right ventricular diameter (RVD), left atrial diameter (LAD), left ventricular (LV) end-systolic (LVESD) and end-diastolic diameter (LVEDD), interventricular septal thickness (IVST), LV posterior wall thickness (LVPWT), ejection fraction (LVEF), LV peak early (E) and late (A) filling velocities, and $\mathrm{E} / \mathrm{A}$ reversal prevalence were measured by transthoracic echocardiography using GE vivid7 full digital color doppler ultrasound diagnostic instrument. ${ }^{12}$

\section{Statistical Analysis}

SPSS (statistical package for social science) 22.0 software (IBM Company, Chicago, Illinois 60606, USA) was used for statistical analysis. The categorical data were expressed as percentage (\%) and compared by chi square test or Fisher exact test. The continuous data were expressed as mean values \pm standard deviation (SD) and evaluated by Mann-Whitney $U$-test or one-way ANOVA (analysis of variance), followed by post hoc analysis using LSD (least significance difference) test for multiple comparisons between the groups. Pearson univariate linear correlation analysis was used to analyze the data between two variables. Dichotomized variate logistic regression was utilized for data analysis. The values of blood pressure were not included in the regression analysis, as considering that blood pressure was collinear with pulse pressure. A 2-tailed value of $P<0.05$ was considered to be statistically significant.

\section{Results}

\section{Clinical Characteristics Corresponding} with eGFR

Compared with the normal eGFR group, patient's age, fasting plasma glucose (FPG), prevalence rate of hypertension and SBP were higher in the mildly declined eGFR group (All $P<0.05$ ); Age, prevalence rate of hypertension and DBP were higher in the moderately declined eGFR group 
(All $P<0.05$ ); Age, FPG, prevalence rate of hypertension, SBP and DBP were higher, while BMI and HDL-C were lower in the severely declined eGFR group (All $P<0.05$ ). Compared with the mildly declined eGFR group, age was older in the moderately declined eGFR group $(P<0.05)$; BMI was lower, while DBP was higher in the severely declined eGFR group (Both $P<0.05$ ). Compared with the moderately declined eGFR group, DBP was higher in the severely declined eGFR group $(P<0.05)$.

Bivariate correlation analysis showed that eGFR was correlated reversely with FPG, prevalence of hypertension and pulse pressure, while it was correlated positively with BMI and HDL-C (All $P<0.05$, Table 1).

\section{Cardiac Remodeling Corresponding with eGFR}

Compared with the normal eGFR group, LAD, IVST, LVPWT and LVESD were larger, while LVEF was lower in the mildly declined eGFR group (All $P<0.05$ ); RAD, LAD, IVST, LVPWT and LVESD were larger, while LVEF was lower in the moderately declined eGFR group (All $P<0.05$ ); RAD, RVD, LAD, IVST, LVPWT, LVESD and LVEDD were larger, while LVEF was lower in the severely declined eGFR group (All $P<0.05$ ). Compared with the mildly declined eGFR group, RAD, LAD, IVST and LVPWT were larger, while LVEF was lower in the moderately declined eGFR group (All $P<0.05$ ); RAD, RVD, LAD, IVST, LVPWT, LVESD and LVEDD were larger, while LVEF was lower in the severely declined eGFR group (All $P<0.05$ ); Compared with the moderately declined eGFR group, RVD, LAD, IVST, LVESD and LVEDD were larger, while LVEF was lower in the severely declined eGFR group (All $P<0.05$ ).

Bivariate correlation analysis showed that eGFR was correlated reversely with RAD, RVD, LAD, IVST, LVPWT, LVESD and LVEDD, while it correlated positively with LVEF (All $P<0.05$, Table 2).

\section{Relationship Between eGFR and Cardiac Remodeling}

Dichotomized variate regression analysis showed that moderate to severe glomerular filtration dysfunction (eGFR $<60 \mathrm{~mL} / \mathrm{min}$ per $1.73 \mathrm{~m}^{2}$ used as the cut-off point) was associated with enlarged left-side atrioventricular chambers, increased ventricular wall thickness, decreased cardiac function and dilated right ventricle (All $P<0.05$, Table 3 ), after adjustment of patient's age, BMI, FPG, HDL-C, hypertension and pulse pressure.

\section{Discussion}

The present study shows that moderate to severe reduction of glomerular filtration function (eGFR $<60 \mathrm{~mL} /$ min per $1.73 \mathrm{~m}^{2}$ ) was found to be associated with enlarged left-side cardiac chambers, increased ventricular wall thickness, decreased cardiac function and dilated right ventricle, after adjustment of patient's age, BMI, FPG, HDL-C, hypertension and pulse pressure. Glomerular filtration dysfunction is associated with cardiac adverse structural remodeling and dysfunction in menopausal Chinese women with type 2 DM.

Diabetes simultaneously affects both the cardiovascular and renal systems. ${ }^{13}$ Although it is generally believed that kidney disease interplays with cardiovascular disease in type $2 \mathrm{DM}^{13}$ the underlying mechanisms for association of glomerular filtration dysfunction with cardiac adverse structural remodeling and dysfunction (renocardiac or type 4 cardiorenal syndrome) in diabetic patients are as follows: Given that there exists insulin resistance in type $2 \mathrm{DM}$ patients, insulin is not able to fully play its normal physiological roles in helping glucose transport into intracellular organelles for utilization. This greatly limits cells to use glucose for its energy supply, leading to extracellular hyperglycemia, while the cells are forced to use fatty acid oxidation for its energy supply. Then, production of reactive oxygen species is increased, which harms glomerular capillary endothelium and basement membrane. ${ }^{2}$ In addition, hyperglycemia increases formation of advanced glycation end products (AGEs). Through binding its specific receptors on cellular surface and activating intracellular signal pathways, AGEs increase oxygen free radicals, peroxidation stress and inflammatory reactions, ${ }^{2}$ and leading to glomerular filtration dysfunction and diabetic nephropathy with consequent retention of water and sodium in blood circulation. Furthermore, due to uremic toxins and nephropathy associated dyspepsia, reduced erythropoietin secretion and anemia occur. $^{14}$ Meanwhile, diabetes-associated atherosclerotic stenosis results in decreased supply of blood flow to the juxtaglomerular apparatus. Consequently, sympathetic nervous and renin angiotensin aldosterone systems are over-activated. ${ }^{15,16}$ Then, cardiac adverse structural remodeling and dysfunction ensue due to the overload of cardiac volume and pressure, which resulted from diabetic nephropathy. ${ }^{14}$ Moreover, recent studies have highlighted that arterial inflammation mediated by 


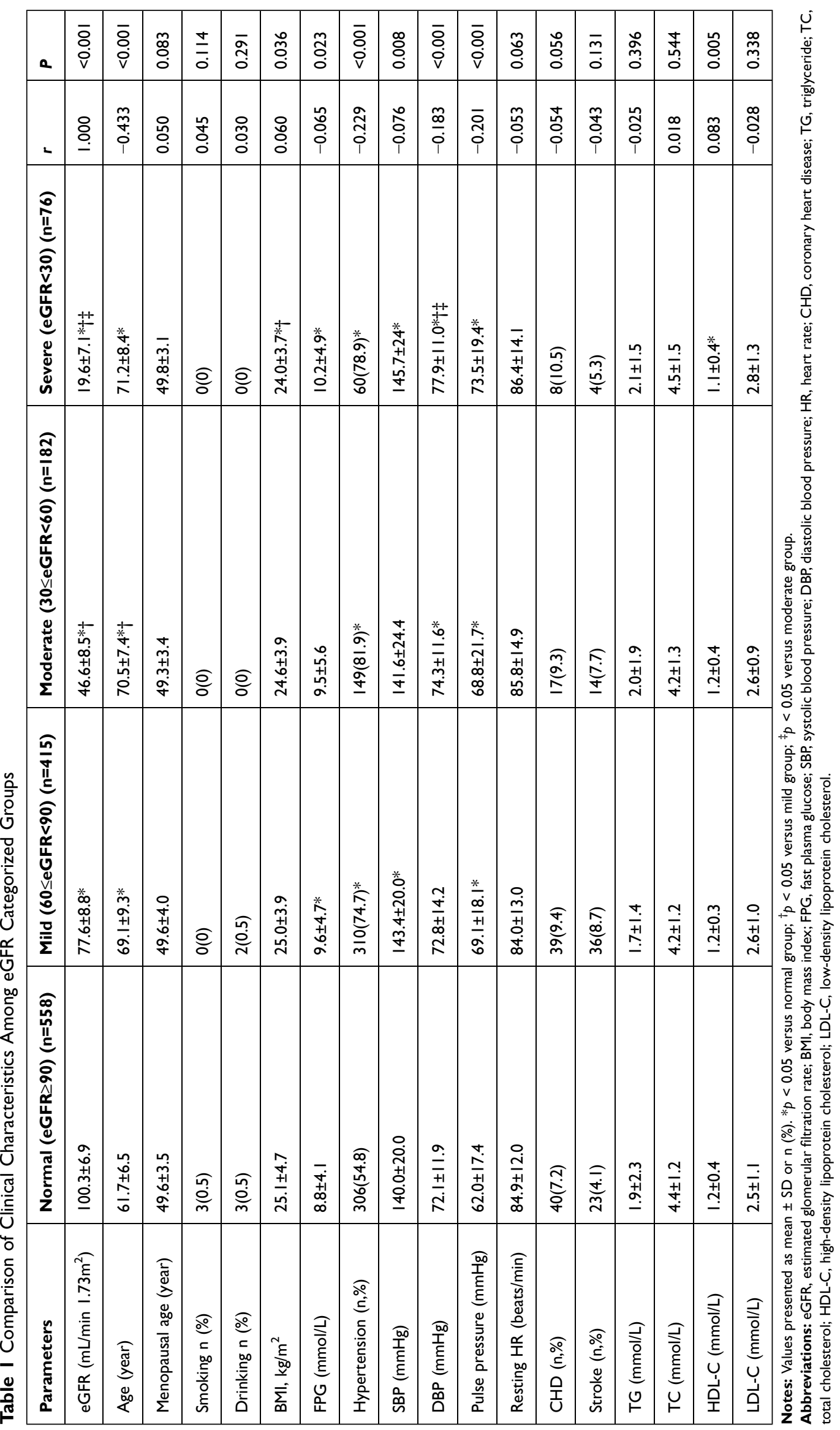


Table 2 Comparison of Echocardiographic Parameters Among eGFR Categorized Groups

\begin{tabular}{|c|c|c|c|c|c|c|}
\hline Parameters & $\begin{array}{l}\text { Normal }(e G F R \geq 90) \\
(n=558)\end{array}$ & $\begin{array}{l}\text { Mild }(60 \leq e G F R<90) \\
(n=4 \mid 5)\end{array}$ & $\begin{array}{l}\text { Moderate }(30 \leq e G F R<60) \\
(n=182)\end{array}$ & $\begin{array}{l}\text { Severe }(e G F R<30) \\
(n=76)\end{array}$ & $r$ & $P$ \\
\hline RAD (mm) & $31.7 \pm 2.9$ & $31.9 \pm 3.2$ & $32.5 \pm 3.5 * \dagger$ & $32.8 \pm 3.8 * \dagger$ & -0.117 & $<0.001$ \\
\hline RVD (mm) & $18.4 \pm 1.9$ & $18.5 \pm 1.6$ & $18.5 \pm 2.1$ & $19.1 \pm 2.1 * \dagger \neq$ & -0.070 & 0.012 \\
\hline LAD (mm) & $29.0 \pm 3.5$ & $29.9 \pm 3.9 *$ & $30.6 \pm 4.0 * \dagger$ & $31.9 \pm 4.8^{*} \dagger \ddagger$ & -0.214 & $<0.001$ \\
\hline IVST (mm) & $10.4 \pm 1.2$ & $10.7 \pm 1.3^{*}$ & $11.0 \pm 1.3^{*} \dagger$ & $11.3 \pm 1.4^{*}+\ddagger$ & -0.239 & $<0.001$ \\
\hline LVPWT (mm) & $10.1 \pm 1.1$ & $10.4 \pm 1.1 *$ & $10.7 \pm 1.2 * \dagger$ & $10.9 \pm 1.3^{*} \dagger$ & -0.233 & $<0.001$ \\
\hline LVESD (mm) & $29.7 \pm 3.4$ & $30.2 \pm 3.2^{*}$ & $30.3 \pm 3.5^{*}$ & $31.8 \pm 4.3^{*} \dagger \ddagger$ & -0.147 & $<0.001$ \\
\hline LVEDD (mm) & $46.2 \pm 4.1$ & $46.7 \pm 4.0$ & $46.5 \pm 4.5$ & $47.9 \pm 4.7^{*} \dagger \ddagger$ & -0.088 & 0.002 \\
\hline LVEF (\%) & $65.4 \pm 4.9$ & $64.7 \pm 4.6^{*}$ & $63.7 \pm 5.2 * \dagger$ & $62.3 \pm 6.2^{*} \dagger \ddagger$ & 0.167 & $<0.001$ \\
\hline $\mathrm{E} / \mathrm{A}<\mathrm{I}(\mathrm{n}, \%)$ & $382(68.5)$ & 297 (7I.6) & I 36 (74.7) & $59(77.6)$ & -0.058 & 0.053 \\
\hline
\end{tabular}

Notes: Values presented as mean \pm SD. ${ }^{*} p<0.05$ versus normal group; ${ }^{\dagger} p<0.05$ versus mild group; ${ }^{\ddagger} p<0.05$ versus moderate group.

Abbreviations: eGFR, estimated glomerular filtration rate $\left(\mathrm{mL} / \mathrm{min} 1.73 \mathrm{~m}^{2}\right)$; RAD, right atrial diameter; RVD, right ventricular diameter; LAD, left atrial diameter; IVST, interventricular septal thickness; LVPWT, left ventricular posterior wall thickness; LVESD, left ventricular end-systolic diameter; LVEDD, left ventricular end-diastolic diameter; LVEF, left ventricular ejection fraction; E/A, peak early (E)/late (A) filling velocities.

Table 3 Regression Analysis of Parameters Associated with eGFR $<60$

\begin{tabular}{|c|c|c|c|c|c|}
\hline & $\boldsymbol{\beta}$ & SE & Wald & $\mathbf{P}$ & OR (95\% CI) \\
\hline Age & 0.059 & 0.010 & 34.935 & $<0.001$ & I.06I (I.040 I.082) \\
\hline BMI & -0.041 & 0.023 & 3.331 & 0.068 & $0.959(0.918 \sim 1.003)$ \\
\hline FPG & 0.023 & 0.016 & 2.655 & 0.156 & $1.023(0.991 \sim 1.056)$ \\
\hline Hypertension & -0.356 & 0.213 & 2.806 & 0.094 & $0.700(0.46 \mathrm{I} \sim 1.062)$ \\
\hline Pulse pressure & 0.003 & 0.004 & 0.362 & 0.547 & $\mathrm{I} .003(0.994 \sim 1.0 \mathrm{II})$ \\
\hline HDL-C & -0.417 & 0.228 & 3.343 & 0.067 & $0.659(0.422 \sim 1.030)$ \\
\hline RAD & 0.042 & 0.028 & 2.312 & 0.128 & $1.043(0.988 \sim 1.102)$ \\
\hline RVD & -0.120 & 0.050 & 5.705 & 0.017 & $0.887(0.804 \sim 0.979)$ \\
\hline LAD & 0.057 & 0.026 & 4.795 & 0.029 & $1.059(1.006 \sim 1.114)$ \\
\hline IVST & 0.226 & 0.102 & 5.565 & 0.019 & I.026 (0.84I I.253) \\
\hline LVPWT & 0.264 & 0.115 & 5.408 & 0.020 & $1.302(1.042 \sim 1.627)$ \\
\hline LVSD & -0.279 & 0.121 & 5.287 & 0.021 & $0.757(0.597 \sim 0.960)$ \\
\hline LVDD & 0.176 & 0.081 & 4.773 & 0.029 & $1.192(1.018 \sim 1.396)$ \\
\hline LVEF & -0.156 & 0.043 & 13.185 & $<0.001$ & $0.856(0.786 \sim 0.931)$ \\
\hline
\end{tabular}

Abbreviations: eGFR, estimated glomerular filtration rate $\left(\mathrm{mL} / \mathrm{min}\right.$ per $\left.1.73 \mathrm{~m}^{2}\right) ; \beta$, regression coefficient; $\mathrm{SE}$, standard error; Wald, Chi square value; $\mathrm{Cl}$, confidence interval; OR, odds ratio; BMI, body mass index; FPG, fast plasma glucose; HDL-C, high-density lipoprotein cholesterol; RAD, right atrial diameter; RVD, right ventricular diameter; LAD, left atrial diameter; IVST, interventricular septal thickness; LVPWT, left ventricular posterior wall thickness; LVESD, left ventricular end-systolic diameter; LVEDD, left ventricular end-diastolic diameter; LVEF, left ventricular ejection fraction.

chronic kidney disease leads to arterial stiffness, which is considered as an important mechanism for the cardiac adverse remodeling in type $2 \mathrm{DM}^{17}$
Physiological menopause is a landmark event for women's aging. Studies have found that menopause increases the risk of cardiovascular mortality in females. ${ }^{18,19}$ The incidence of type 
$2 \mathrm{DM}$ is increased abruptly when people enter into old age. ${ }^{20}$ The cardiovascular events and mortality are more higher in postmenopausal women with type 2 DM than those of diabetic men at the same age. ${ }^{7,8}$ It suggests that combination of menopause with type 2 DM could greatly increase cardiovascular events and death in postmenopausal diabetic women as compared the women without menopause and type $2 \mathrm{DM}^{21}$ The increased cardiovascular events and death in menopausal diabetic women are usually ascribed to diabetes-associated cardiomyopathy and coronary atherosclerotic disease., ${ }^{7,8,21}$ Nevertheless, previous studies have proved that the increased risk of cardiovascular events and mortality in diabetic patients were ascribed to glomerular filtration dysfunction as well. ${ }^{3,4,22}$ Several studies reported that reduced eGFR was associated with cardiac structural remodeling and dysfunction in diabetic adults. ${ }^{5,6,23,24}$

Although, in this study, BMI, FPG, HDL-C, hypertension and pulse pressure (an useful indicator for the assessment of arterial stiffness) were not found to be independently associated with cardiac structural changes and dysfunction in type 2 DM, our present study replenishes that glomerular filtration dysfunction is associated with cardiac adverse structural remodeling and dysfunction in menopausal Chinese women with type 2 DM. Nevertheless, the cardiac LV diastolic dysfunction defined by E/A reversal was not found to be significantly different among eGFR categorized groups. The E/A reversal may not precisely reflect the LV diastolic dysfunction. ${ }^{25}$

Our results suggest that earlier identification and intervention of glomerular filtration dysfunction may be very important in the prevention from the cardiac adverse structural remodeling and dysfunction in type 2 DM. However, intensive glucose control is not advised in the patient with diabetic nephropathy. ${ }^{26}$

A major limitation of the present study is that the data was obtained from a cross-sectional study. Although regression analyses were performed, the effects of other possible confounding factors should be taken into consideration. Therefore, large prospective studies would be needed to reconfirm the observed findings. The study was carried on menopause women, its results should be cautiously extrapolated to other populations.

\section{Conclusion}

Glomerular filtration dysfunction is associated with cardiac adverse structural remodeling and dysfunction in menopausal Chinese women with type 2 DM, after adjustment of patient's BMI, FPG, HDL-C, hypertension and pulse pressure.

\section{Acknowledgments}

This study was supported by the Natural Science Research Fund of the Medical Science \& Technology Research Fund of Health Bureau of Chongqing City, China (No. 04-2-154 and No. 2009-2-290) and Chongqing Science \& Technology Commission in Chongqing City, China (CSTC, No. 2007BB5276).

\section{Disclosure}

The authors report no conflicts of interest in this work.

\section{References}

1. Walker AM, Cubbon RM. Sudden cardiac death in patients with diabetes mellitus and chronic heart failure. Diab Vasc Dis Res. 2015;12(4):228-233. doi:10.1177/1479164115573225

2. Joubert M, Manrique A, Cariou B, et al. Diabetes-related cardiomyopathy: the sweet story of glucose overload from epidemiology to cellular pathways. Diabetes Metab. 2019;45(3):238-247. doi:10.1016/j.diabet.2018.07.003

3. Nag S, Bilous R, Kelly W, et al. All-cause and cardiovascular mortality in diabetic subjects increases significantly with reduced estimated glomerular filtration rate (eGFR): 10 years' data from the South Tees Diabetes Mortality study. Diabet Med. 2007;24(1):10-17. doi:10.1111/j.1464-5491.2007.02023.x

4. Matsushita K, Coresh J, Sang Y, et al. Estimated glomerular filtration rate and albuminuria for prediction of cardiovascular outcomes: a collaborative meta-analysis of individual participant data. Lancet Diabetes Endocrinol. 2015;3(7):514-525. doi:10.1016/S2213-8587(15)00040-6

5. Wu PY, Huang JC, Chen SC, et al. Type 2 diabetes mellitus-related changes in left ventricular structure and function in patients with chronic kidney disease. Oncotarget. 2018;9(18):14661-14668. doi:10.18632/oncotarget.24482

6. Chen SC, Chang JM, Liu WC, et al. Stepwise increases in left ventricular mass index and decreases in left ventricular ejection fraction correspond with the stages of chronic kidney disease in diabetes patients. Exp Diabetes Res. 2012;2012:789325. doi:10.1155/2012/789325

7. Ma Y, Persuitte GM, Andrews C, et al. Impact of incident diabetes on atherosclerotic cardiovascular disease according to statin use history among postmenopausal women. Eur J Epidemiol. 2016;31 (8):747-761. doi:10.1007/s 10654-016-0153-7

8. Maiello M, Zito A, Cecere A, et al. Left ventricular diastolic dysfunction in normotensive postmenopausal women with type 2 diabetes mellitus. Cardiol J. 2017;24(1):51-56. doi:10.5603/CJ.a2016.0064

9. Alberti KG, Zimmet PZ. Definition, diagnosis and classification of diabetes mellitus and its complications. Part 1: diagnosis and classification of diabetes mellitus provisional report of a WHO consultation. Diabet Med. 1998;15(7):539-553. doi:10.1002/(SICI) 1096-9136(199807)15:7<539::AID-DIA668>3.0.CO;2-S

10. Inker LA, Schmid $\mathrm{CH}$, Tighiouart $\mathrm{H}$, et al. Estimating glomerular filtration rate from serum creatinine and cystatin C. $N$ Engl J Med. 2012;367(1):20-29. doi:10.1056/NEJMoa1114248

11. Liu C, Li G, Laukkanen JA, et al. Overweight and obesity are associated with cardiac adverse structure remodeling in Chinese elderly with hypertension. Sci Rep. 2019;9(1):17896. doi:10.1038/ s41598-019-54359-9

12. Lang RM, Badano LP, Mor-Avi V, et al. Recommendations for cardiac chamber quantification by echocardiography in adults: an update from the American Society of Echocardiography and the European Association of Cardiovascular Imaging. Eur Heart J Cardiovasc Imaging. 2015;16 (3):233-270. doi:10.1093/ehjci/jev014 
13. Rodriguez E, Arias-Cabrales C, Pascual J. Diabetes mellitus: a single cardiorenal syndrome umbrella. Clin Kidney J. 2020;13(1):14-16. doi:10.1093/ckj/sfz161

14. Kingma JG, Simard D, Rouleau JR. Renocardiac syndromes: physiopathology and treatment stratagems. Can $J$ Kidney Health Dis. 2015;2:41. doi:10.1186/s40697-015-0075-4

15. Komici K, Femminella GD, de Lucia C, et al. Predisposing factors to heart failure in diabetic nephropathy: a look at the sympathetic nervous system hyperactivity. Aging Clin Exp Res. 2019;31 (3):321-330. doi:10.1007/s40520-018-0973-2

16. Lozano-Maneiro L, Puente-Garcia A. Renin-angiotensin-aldosterone system blockade in diabetic nephropathy. Present evidences. J Clin Med. 2015;4(11):1908-1937. doi:10.3390/jcm4111908

17. Zanoli L, Lentini $\mathrm{P}$, Briet $\mathrm{M}$, et al. Arterial stiffness in the heart disease of CKD. $J$ Am Soc Nephrol. 2019;30(6):918-928. doi:10.1681/ASN.2019020117

18. Bertoia ML, Allison MA, Manson JE, et al. Risk factors for sudden cardiac death in post-menopausal women. $\mathrm{J}$ Am Coll Cardiol. 2012;60(25):2674-2682. doi:10.1016/j.jacc.2012.09.031

19. Machi JF, Dias Dda S, Freitas SC, et al. Impact of aging on cardiac function in a female rat model of menopause: role of autonomic control, inflammation, and oxidative stress. Clin Interv Aging. 2016;11:341-350. doi:10.2147/CIA.S88441

20. Zhao Q, Laukkanen JA, Li Q, et al. Body mass index is associated with type 2 diabetes mellitus in Chinese elderly. Clin Interv Aging. 2017;12:745-752. doi:10.2147/CIA.S130014
21. Zhou H, Zhang C, Ni J, et al. Prevalence of cardiovascular risk factors in non-menopausal and postmenopausal inpatients with type 2 diabetes mellitus in China. BMC Endocr Disord. 2019;19(1):98. doi:10.1186/s12902-019-0427-7

22. Amod A, Buse JB, McGuire DK, et al. Glomerular filtration rate and associated risks of cardiovascular events, mortality, and severe hypoglycemia in patients with type 2 diabetes: secondary analysis (DEVOTE 11). Diabetes Ther. 2020;11(1):53-70. doi:10.1007/ s13300-019-00715-x

23. Bayauli MP, Lepira FB, Kayembe PK, et al. Left ventricular hypertrophy and geometry in type 2 diabetes patients with chronic kidney disease. An echocardiographic study. Cardiovasc J Afr. 2012;23 (2):73-77. doi:10.5830/CVJA-2011-028

24. Matsushita K, Ballew SH, Coresh J. Influence of chronic kidney disease on cardiac structure and function. Curr Hypertens Rep. 2015;17(9):581.25. doi:10.1007/s11906-015-0581-x

25. Ozkan H, Akdemir S, Tiryakioglu S, et al. The evaluation of type 2 diabetes mellitus related changes in diastolic dysfunction during exercise using conventional and tissue doppler echocardiography. Cardiol Res. 2015;6(6):346-351. doi:10.14740/cr439w

26. Marso SP, Kennedy KF, House JA, et al. The effect of intensive glucose control on all-cause and cardiovascular mortality, myocardial infarction and stroke in persons with type 2 diabetes mellitus: a systematic review and meta-analysis. Diab Vasc Dis Res. 2010;7 (2):119-130. doi:10.1177/1479164109353367
Clinical Interventions in Aging

\section{Publish your work in this journal}

Clinical Interventions in Aging is an international, peer-reviewed journal focusing on evidence-based reports on the value or lack thereof of treatments intended to prevent or delay the onset of maladaptive correlates of aging in human beings. This journal is indexed on PubMed Central, MedLine, CAS, Scopus and the Elsevier

\section{Dovepress}

Bibliographic databases. The manuscript management system is completely online and includes a very quick and fair peer-review system, which is all easy to use. Visit http://www.dovepress.com/ testimonials.php to read real quotes from published authors. 